RESEARCH HIGHLIGHTS

\section{SIGNAL TRANSDUCTION}

\section{Take it away...}

A crucial aspect of biological signalling is knowing when to stop. The mechanism behind another important aspect - how to stop - has just been discovered in the context of signalling by the epidermal growth factor receptor (EGFR) in Drosophila melanogaster. In Nature, Klein et al. report that the secreted protein Argos functions as an antagonist of EGFR signalling by sequestering the EGF ligand Spitz.

The group had thought that Argos might directly interact with EGFR to stabilize an autoinhibited conformation, so they studied the interactions of Argos, EGFR and Spitz using purified recombinant forms of the proteins in surface-plasmon-resonance studies. Surprisingly, and therefore contrary to their original theory, Argos and EGFR didn't interact. But Argos did interact with Spitz in a 1:1 complex. Argos bound to the EGF-like domain of Spitz, and Spitz bound to the carboxy-terminal part of Argos.

On the basis of these data, Klein et al. proposed that, because Spitz could bind to Argos or EGFR, Argos might function by 'taking' Spitz away from EGFR and blocking its EGFRbinding site - analogous to antagonists of signalling by insulin-like growth factor-1 or bone morphogenetic protein. By adding increasing amounts of Argos to a fixed concentration of free Spitz and testing receptor binding, the authors could show that Argos abolished the Spitz-EGFR interaction. This was most effective when Argos and Spitz were present at the same concentration, consistent with the 1:1 binding ratio.

In a cellular context, adding increasing amounts of Argos progressively decreased the Spitzinduced tyrosine phosphorylation of EGFR. When Klein et al. used fluorescently tagged recombinant proteins to label cell surfaces, they found that Spitz only bound to the surface of cells that were expressing EGFR, and this binding was reduced if Argos was added at the same time or beforehand. However, Argos couldn't displace Spitz from preformed Spitz-EGFR complexes at the cell surface, which led the authors to conclude that Argos can't reverse EGFR activation once it's started.

So these findings go against previous suggestions that Argos is an antagonistic EGFR ligand. Furthermore, this mechanism of inhibition is more consistent with that of other signalling antagonists that sequester stimulatory ligands. As Argos is a Spitz-induced Spitz antagonist, the authors suggest that local production of Argos might effectively limit longer-range signalling by Spitz during D. melanogaster development.

Katrin Bussell

\section{(2) References and links}

ORIGINAL RESEARCH PAPER Klein, D. E. et al. Argos inhibits epidermal growth factor receptor signalling by ligand sequestration. Nature $\mathbf{4 3 0}$, 1040-1044 (2004)

FURTHER READING Jorissen, R. N. et al. Epidermal growth factor: mechanisms of activation and signalling. Exp. Cell Res. 284, 31-53 (2003) WEB SITE

Mark Lemmon's laboratory:

http://www.med.upenn.edu/camb/faculty/cgc/ lemmon.html

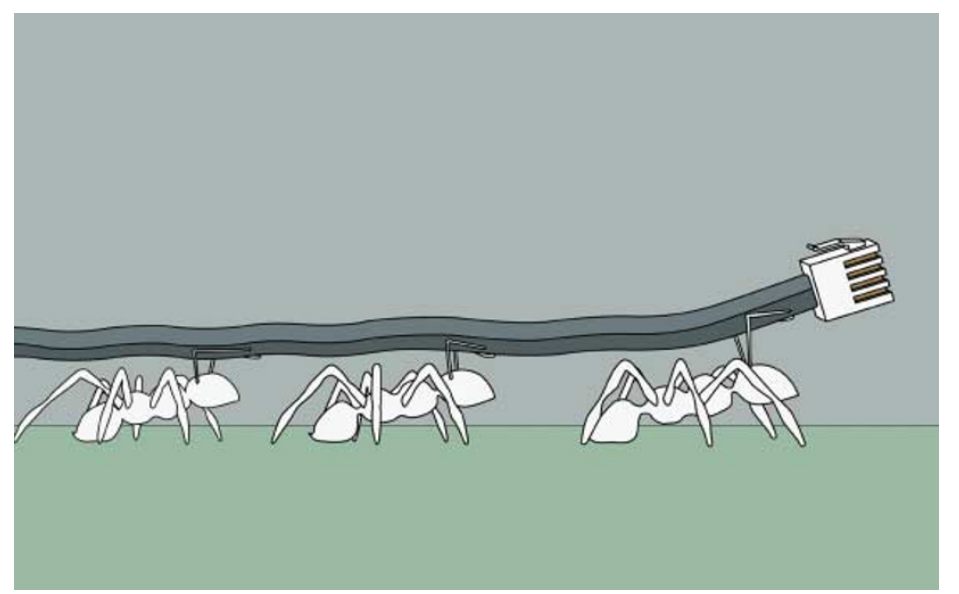

\section{IN BRIEF}

\section{PROTEOMICS}

Temporal analysis of phosphotyrosine-dependent signaling networks by quantitative proteomics.

Blagoev, B. et al. Nature Biotech. 22, 1139-1145 (2004)

The authors developed a proteomics approach that enabled the global, time-dependent analysis of the epidermal growth factor receptor (EGFR) phosphotyrosine proteome. Cells were cultured in medium that contained one of three different isoforms of arginine, stimulated with EGF for defined times and then lysed. A higher-resolution profile was obtained by repeating the procedure with three additional time points. Tyrosine-phosphorylated proteins were affinity purified and then subjected to tryptic digestion and mass spectrometry. At each time point, the peak intensities indicated the amount of the tyrosine-phosphorylated protein, or proteins that bound to it. This approach identified several proteins that weren't previously associated with EGFR signalling, and the authors envisage the use of such temporal analysis for other tyrosine-mediated signalling pathways.

\section{NUCLEOCYTOPLASMIC TRANSPORT}

\section{Exportin 7 defines a novel general nuclear export} pathway.

Mingot, J.-M. et al. EMBO J. 23, 3227-3236 (2004)

Until now, the function of RanBP16, a member of the importin- $\beta$ family of nuclear-transport receptors that mediate nucleocytoplasmic transport, has been elusive. But Mingot et al. have shown that RanBP16 is a nuclear-export receptor that excludes the GTPase-activating protein $\mathrm{p} 50 \mathrm{RhoGAP}$ and the scaffold protein 14-3-3 $\sigma$ from the nucleus - thereby restricting their function to the cytoplasm. The authors therefore renamed RanBP16 'exportin 7'. Analysis of the nuclear-export signals of these proteins showed that folded motifs could be recognized, and that there was a requirement for basic residues - which is not the case for the nuclear-export receptor CRM1.

\section{CYTOKINESIS}

Cytokinesis monitoring during development: rapid pole-to-pole shuttling of a signaling protein by localized kinase and phosphatase in Caulobacter.

Matroule, J.-Y. et al. Cell 118, 579-590 (2004)

Asymmetric cell division generates cell diversity, but cells should not be allowed to differentiate until division has finished. When studying how such regulation might occur in the bacterium Caulobacter crescentus, Matroule et al. found that the signalling protein DivK - which is dynamically localized at both poles by a 'ping-pong' mechanism before division — is released from the flagellar pole after cytokinesis is completed. Development then follows in the flagellar progeny. The bipolar distribution usually occurs through the activities of DivJ (a kinase) and PleC (a phosphatase) at opposite ends of the bacterium. DivJ induces DivK localization at the flagellar pole, from which it is released by PleC. Cytokinesis blocks this exchange of DivK. 